\title{
Soluble Star-shaped Molecules based on Thiophene Derivatives as Organic Semiconductors for Field-Effect Transistor Applications
}

Kyung Hwan Kim, ${ }^{\dagger}$ Zhenguo Chi, ${ }^{\dagger}$ Min Ju Cho, ${ }^{\dagger}$ Jung-Il Jin, ${ }^{\dagger}$ Mi Yeon Cho, ${ }^{\ddagger}$ Su Jin

Kim, ${ }^{\ddagger}$ Jin-soo Joo ${ }^{\ddagger}$, Dong Hoon Choi ${ }^{+*}$

${ }^{\dagger}$ Department of Chemistry, Center for Electro- \& Photo-responsive Molecules,

Korea University, Seoul 136-701, South Korea

${ }^{\ddagger}$ Department of Physics, Korea University, Seoul 136-701, South Korea

*Corresponding author (hhchoi8803@korea.ac.kr)

TITLE RUNNING HEAD: Soluble Star-shaped Semiconducting Molecules

Synthesis and spectroscopic characterization

General: All commercially available starting materials were purchased from Aldrich, or

ACROS Co. and used without further purification unless otherwise stated. HPLC grade tetrahydrofuran (THF) and diethyl ether were purchased from Samkyung Chemical Co. and distilled from sodium and benzophenone immediately before use. HPLC grade 
methylene chloride (MC) was also purchased from Fisher and distilled from $\mathrm{CaH}$ prior to use. All reactions were performed under an argon atmosphere unless otherwise stated.

\section{Synthesis}

Compounds 1, 2, 5, 6, 9, 10, and [1,2,4,5-tetra-(diethoxy-phosphorylmethyl)-benzyl]phosphonic acid diethyl ester as a core unit were synthesized by following the literature method and a modified method..$^{9-15}$

\section{5'-Hexyl-[2,2']bithiophenyl-5-carbaldehyde, 3}

In a 100mL, oven dried, mag.-stirred round bottom flask (RBF), 2 (4.00 g, $16.00 \mathrm{mmol})$ was dissolved in freshly distilled THF $(50 \mathrm{~mL})$. The solution was then cooled to $-78^{\circ} \mathrm{C}$. n-BuLi (8.00 mL, $16.00 \mathrm{mmol}, 2.5 \mathrm{M}$ sol'n. in hexane) was then added dropwise over 15 minutes. The mixture was allowed to stir for 0.5 hour and then quenched with dry DMF (1.17 g, $16.00 \mathrm{mmol})$. Reaction was continued under stirring for $3 \mathrm{~h}$ at room temperature and then $200 \mathrm{~mL}$ distilled water was added into it. The organic layer was extracted with MC (3 x 100ml). The organics were then dried over $\mathrm{MgSO}_{4}$, filtered, and the solvent was concentrated. The resulting crude product was then purified by silica gel 
column chromatography (eluent; EA: Hexane=1:5 v/v) to yield $3.23 \mathrm{~g}(73 \%)$ of 5'hexyl-[2,2']bithiophenyl-5-carbaldehyde, 3.

${ }^{1} \mathrm{H}-\mathrm{NMR}\left(400 \mathrm{MHz}, \mathrm{CDCl}_{3}\right.$ ): $\delta(\mathrm{ppm}) 9.81$ (s, 1H), 7.62 (d, $\left.J=4.0 \mathrm{~Hz}, 1 \mathrm{H}\right), 7.16$ (d, $J=$ $4.0 \mathrm{~Hz}, 1 \mathrm{H}), 7.14(\mathrm{~d}, J=4.0 \mathrm{~Hz}, 1 \mathrm{H}), 6.72(\mathrm{~d}, J=4.0 \mathrm{~Hz}, 1 \mathrm{H}), 2.79(\mathrm{t}, 2 \mathrm{H}), 1.62 \sim 1.70(\mathrm{~m}$, 2H), 1.25 1.39 (m, 6H), 0.87 (t, 3H)

${ }^{13} \mathrm{C}-\mathrm{NMR}\left(125 \mathrm{MHz}, \mathrm{CDCl}_{3}\right): \delta(\mathrm{ppm})$ 182.72, 148.95, 148.16, 141.17, 137.77, 133.59, $126.29,125.69,123.63,31.74,31.71,30.49,28.95,22.79,14.31$

EA analysis calcd for $\mathrm{C}_{15} \mathrm{H}_{18} \mathrm{OS}_{2}$; C, 64.71; $\mathrm{H}, 6.52$; $\mathrm{S}, 23.03$, found ; 64.78; $\mathrm{H}, 6.43$; $\mathrm{S}$, 22.68

HRMS $m / z$ calcd for $\mathrm{C}_{15} \mathrm{H}_{18} \mathrm{OS}_{2}$. [Na] $]^{+}, 301.07$, found 301.16

\section{1,2,4,5-Tetra(5'-hexyl-[2,2']bithiophenyl-5-vinyl)-benzene, 4}

An oven dried, mag.-stirred, $100 \mathrm{~mL}$ RBF was charged with a solution of 3 (3.00 g, $10.8 \mathrm{mmol}$ ) and [1,2,4,5-tetra-(diethoxy-phosphorylmethyl)-benzyl]-phosphonic acid diethyl ester (1.83 g, $2.70 \mathrm{mmol})$ in $50 \mathrm{~mL}$ freshly distilled THF. The reaction was allowed to stir for $0.5 \mathrm{~h}$ followed by addition of potassium tert-butoxide (1.80 g, 16.2 mmole). After completing the reaction, the solution was poured into ethanol to collect the precipitates. yield $2.57 \mathrm{~g}(82 \%)$. 
${ }^{1} \mathrm{H}-\mathrm{NMR}\left(400 \mathrm{MHz}, \mathrm{CDCl}_{3}\right.$ ): $\delta(\mathrm{ppm}) 7.55$ (s, 2H), 7.10 (d, $\left.J=16.0 \mathrm{~Hz}, 4 \mathrm{H}\right), 7.06$ (d, $J$ $=16.0 \mathrm{~Hz}, 4 \mathrm{H}), 6.98(\mathrm{~d}, J=4.0 \mathrm{~Hz}, 4 \mathrm{H}), 6.97(\mathrm{~d}, J=4.0 \mathrm{~Hz}, 4 \mathrm{H}), 6.94(\mathrm{~d}, J=4.0 \mathrm{~Hz}, 4 \mathrm{H})$ $6.65(\mathrm{~d}, J=4.0 \mathrm{~Hz}, 4 \mathrm{H}) 2.78(\mathrm{t}, 8 \mathrm{H}), 1.64 \sim 1.71(\mathrm{~m}, 8 \mathrm{H}), 1.24 \sim 1.42(\mathrm{~m}, 24 \mathrm{H}), 0.89(\mathrm{t}$, $12 \mathrm{H})$

${ }^{13} \mathrm{C}-\mathrm{NMR}\left(125 \mathrm{MHz}, \mathrm{CDCl}_{3}\right): \delta(\mathrm{ppm})$ 145.86, 141.08, 137.78, 137.16, 134.95, 127.61, 125.02, 124.97, 123.64, 123.59, 123.42, 122.46, 31.72, 31.68, 30.32, 28.91, 22.72, 14.22

EA analysis calcd for $\mathrm{C}_{70} \mathrm{H}_{78} \mathrm{~S}_{8} ; \mathrm{C}, 71.50 ; \mathrm{H}, 6.69 ; \mathrm{S}, 21.81$, found: $\mathrm{C}, 71.40 ; \mathrm{H}, 6.61 ; \mathrm{S}$, 21.90

MALDI exact mass calcd for: $\mathrm{C}_{70} \mathrm{H}_{78} \mathrm{~S}_{8}$. [M] $]^{+}, 1173.3791$, found 1173.3991

\section{5-Hexyl-thieno[3,2-b]thiophene-2-carbaldehyde, 7}

The preparation method was the same as that of 3. Instead of 5-hexyl-[2,2']bithiophenyl, 2, 2-hexyl-thieno[3,2-b]thiophene, 6 was employed. The resulting crude product was then purified by silica gel column chromatography (eluent; EA: Hexane=1:5 v/v) to yield $4.56 \mathrm{~g}$ (76\%) of 5-hexyl-thieno[3,2-b]thiophene-2-carbaldehyde. 7.

${ }^{1} \mathrm{H}-\mathrm{NMR}\left(300 \mathrm{MHz}, \mathrm{CDCl}_{3}\right.$ ): $\delta(\mathrm{ppm}) 9.90$ (s, 1H), 7.83 (s, 1H), 7.00 (s, 1H), 2.90 (t, 2H), $1.68 \sim 1.76$ (m, 2H), 1.28 1.40 (m, 6H), 0.88 (t, 3H) 
${ }^{13} \mathrm{C}-\mathrm{NMR}\left(125 \mathrm{MHz}, \mathrm{CDCl}_{3}\right):$ 183.53, 156.46, 146.28, 143.84, 137.64, 129.61, 117.31, 31.82, 31.71, 31.45, 28.94, 22.77, 14.30

EA analysis calcd for $\mathrm{C}_{13} \mathrm{H}_{16} \mathrm{OS}_{2}$; C, 61.86; H, 6.39; S, 25.41, found ; C, 62.40; H, 6.93; S, 24.58

HRMS $m / z$ calcd for $\mathrm{C}_{15} \mathrm{H}_{18} \mathrm{OS}_{2}$. [Na] $]^{+}, 275.10$, found 275.20

\section{1,2,4,5-Tetra(2-Hexyl-5-vinyl-thieno[3,2-b]thiophene)-benzene, 8}

The preparation method was the same as that of 4. Instead of 5'-hexyl[2,2']bithiophenyl-5-carbaldehyde, 3, 5-hexyl-thieno[3,2-b]thiophene-2-carbaldehyde, 7 was employed. After completing the reaction, the solution was poured into ethanol to collect the precipitates. yield $1.71 \mathrm{~g}$ (76 \%).

${ }^{1} \mathrm{H}-\mathrm{NMR}\left(300 \mathrm{MHz}, \mathrm{CDCl}_{3}\right): \delta(\mathrm{ppm}) 7.67$ (s, 2H), $7.22(\mathrm{~d}, J=18.0 \mathrm{~Hz}, 4 \mathrm{H}), 7.17$ (d, $J$ $=18.0 \mathrm{~Hz}, 4 \mathrm{H}), 7.16(\mathrm{~s}, 4 \mathrm{H}), 6.91(\mathrm{~s}, 4 \mathrm{H}), 2.89(\mathrm{t}, 8 \mathrm{H}), 1.69 \sim 1.77(\mathrm{~m}, 8 \mathrm{H}), 1.31 \sim 1.43(\mathrm{~m}$, 24H), $0.91(\mathrm{t}, 12 \mathrm{H})$

${ }^{13} \mathrm{C}-\mathrm{NMR}\left(125 \mathrm{MHz}, \mathrm{CDCl}_{3}\right)$ :149.32, 143.72, 138.00, 137.98, 137.74, 134.52, 125.09, 124.81, 119.20, 116.77, 31.79, 31.70, 31.47, 29.02, 22.80, 14.30

EA analysis calcd for $\mathrm{C}_{62} \mathrm{H}_{70} \mathrm{~S}_{8}$; C, 69.48; H, 6.58; S, 23.93, found: C, 69.66; H, 6.32; S, 23.88 
HRMS $m / z$ calcd for $\mathrm{C}_{62} \mathrm{H}_{70} \mathrm{~S}_{8}$, [M] $]^{+}$1070.32, found 1070.89

\section{6-Hexyl-dithieno[3,2-b;2',3’-d]thiophene-2-carbaldehyde, 11}

The preparation method was the same as that of $\mathbf{3}$. Instead of 2, 6-Hexyl-dithieno[3,2b;2',3'-d]thiophene, 10 was used in this reaction. The resulting crude product was then purified by silica gel column chromatography (eluent; chloroform: Hexane=5:1 v/v) to yield 1.42 g (86\%) of 6-hexyl-dithieno[3,2-b;2’,3’-d]thiophene-2-carbaldehyde, 11.

${ }^{1} \mathrm{H}-\mathrm{NMR}\left(300 \mathrm{MHz}, \mathrm{CDCl}_{3}\right.$ ): $\delta(\mathrm{ppm}) 9.90$ (s, 1H), 7.90 (s, 1H), 7.01 (s, 1H), 2.90 (t, 2H), $1.76 \sim 1.68(\mathrm{~m}, 2 \mathrm{H}), 1.42 \sim 1.28(\mathrm{~m}, 6 \mathrm{H}), 0.88(\mathrm{t}, 3 \mathrm{H})$

${ }^{13} \mathrm{C}-\mathrm{NMR}$ (125 MHz, $\mathrm{CDCl}_{3}$ ): 183.01, 152.20, 145.95, 143.03, 140.11, 138.67, 130.60, 128.84, 118.05, 31.69, 31.55, 28.86, 28.86, 22.72, 14.24

EA analysis calcd for $\mathrm{C}_{15} \mathrm{H}_{16} \mathrm{OS}_{3} ; \mathrm{C}, 58.40 ; \mathrm{H}, 5.23$; S, 31.18 ; found ; C, 58.35; H, 5.17; S, 31.60

HRMS m/z calcd for $\mathrm{C}_{15} \mathrm{H}_{16} \mathrm{OS}_{3}, \quad[\mathrm{Na}]^{+} 331.03$ found 331.09

\section{1,2,4,5-Tetra(6-hexyl-2-vinyl-dithieno[3,2-b;2',3'-d]thiophene)-benzene, 12}

The preparation method was the same as that of 4. Instead of 5'-hexyl[2,2']bithiophenyl-5-carbaldehyde, 3, 6-hexyl-dithieno[3,2-b;2',3'-d]thiophene-2- 
carbaldehyde, $\mathbf{1 1}$ was used in this reaction. After completing the reaction, the solution was poured into ethanol to collect the precipitates. yield $1.09 \mathrm{~g}$ (86 \%).

${ }^{1} \mathrm{H}-\mathrm{NMR}\left(300 \mathrm{MHz}, \mathrm{CDCl}_{3}\right.$ ): $\delta(\mathrm{ppm}) 7.47$ (s, 2H), 7.11 (s, 4H), 7.10 (d, $J=17.0 \mathrm{~Hz}$, 4H), 7.00 (d, $J=17.0 \mathrm{~Hz}, 4 \mathrm{H}), 6.98$ (s, 4H), 2.90 (t, 8H), 1.77 1.72 (m, 8H), 1.44 1.35 (m, 24H), $0.92(\mathrm{t}, 12 \mathrm{H})$

${ }^{13}$ C-NMR (125 MHz, $\mathrm{CDCl}_{3}$ ): 148.15, 143.34, 141.40, 140.45, 134.47, 130.41, 129.30, 124.79, 124.73, 120.37, 117.72, 31.83, 31.49, 31.15, 29.03, 22.83, 14.33.

EA analysis calcd for $\mathrm{C}_{70} \mathrm{H}_{70} \mathrm{~S}_{12}$; C, 64.87; H, 5.44; S, 29.69 found: C, 64.77 H, 5.39; S, 29.63

HRMS $m / z$ calcd for $\mathrm{C}_{70} \mathrm{H}_{70} \mathrm{~S}_{12}$. [M] ${ }^{+}$1294.21, observed 1294.74 